Gut, 1986, 27, 1394-1398

Case report

\title{
Collagenous colitis associated with small intestinal villous atrophy
}

\author{
I HAMILTON, S SANDERS, D HOPWOOD, AND I A D BOUCHIER \\ From the University Department of Medicine and Department of Pathology, Ninewells Hospital and Medical \\ School, Dundee, Scotland
}

SUMmaRY Two patients diagnosed as having small intestinal hyperplastic villous atrophy and being treated with a gluten free diet were investigated because of persistent watery diarrhoea. Both were found to have collagenous colitis. Previous reports of this condition have emphasised the presence of normal small intestinal mucosal architecture and the association of collagenous colitis with intestinal villous atrophy has not previously been reported. Both cases responded to oral steroid therapy, but not to other previously recommended treatment regimens.

Collagen deposition in the lamina propria of the small intestine is recognised as a feature of coeliac disease, which usually regresses after treatment with

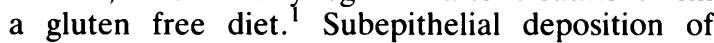
collagen in the colon has also been described. Thickening of the colonic basement membrane is associated with metaplastic colonic polyps, ${ }^{2}$ colonic carcinoma, and inflammatory bowel disease, ${ }^{3}$ but significant collagen deposition within the lamina propria of the colon is characteristic of collagenous colitis, an infrequent but increasingly recognised cause of chronic or recurrent watery diarrhoea. The pathogenesis of collagenous colitis and its association with other gastrointestinal or systemic diseases has not been elucidated, and there is no recognised association with small intestinal disease, jejunal biopsy being normal in all previously reported cases in whom it has been done. ${ }^{410}$

We describe two patients with coeliac disease in whom diarrhoea persisted or recurred despite treatment with a gluten free diet, and in whom investigations revealed the coexistence of collagenous colitis.

\section{Case reports}

CASE 1

A 45 year old woman presented in 1967 with a

Address for correspondence: Dr I Hamilton, Department of Medicine. Ninewells Hospital and Medical School, Dundec DD1 9SY. Scotland.

Received for publication 21 February 1986. history of longstanding diarrhoea. She was found to have a hypochromic microcytic anaemia, low serum folate, and low serum $B_{12}$. Jejunal biopsy showed subtotal villous atrophy and she made a good clinical response to a gluten free diet. In 1981 she complained of intermittent severe watery diarrhoea despite rigid adherence to a gluten free diet. Investigations revealed normal full blood count, serum urea and electrolytes, liver and thyroid function tests, immunoglobulins and iron. No pathogenic organisms or parasites were isolated from her stools. Small and large bowel barium studies were normal, faecal fat was not raised and pancreatic function tests were normal. Jejunal biopsy revealed a normal mucosal architecture with no increase in inflammatory cells and no evidence of parasites. Sigmoidoscopy was normal and rectal biopsy showed only a mild, non-specific chronic proctitis. Her diarrhoea resolved spontaneously.

In 1985 she was again admitted for investigation of profuse watery diarrhoea. Haematological and biochemical investigations were again normal, there were no pathogens present in the stool, ${ }^{14} \mathrm{C}$ glycocholate breath test was normal and jejunal biopsy was again within normal limits (Fig. 1). At colonoscopy the colonic mucosa looked pale but was otherwise normal. Multiple biopsies showed the appearances of collagenous colitis (Fig. 2).

Symptoms failed to respond to treatment with metronidazole, salazopyrin or rectal steroids, but oral prednisolone $30 \mathrm{mg}$ daily induced a prompt remission. Within two weeks of instituting treatment 

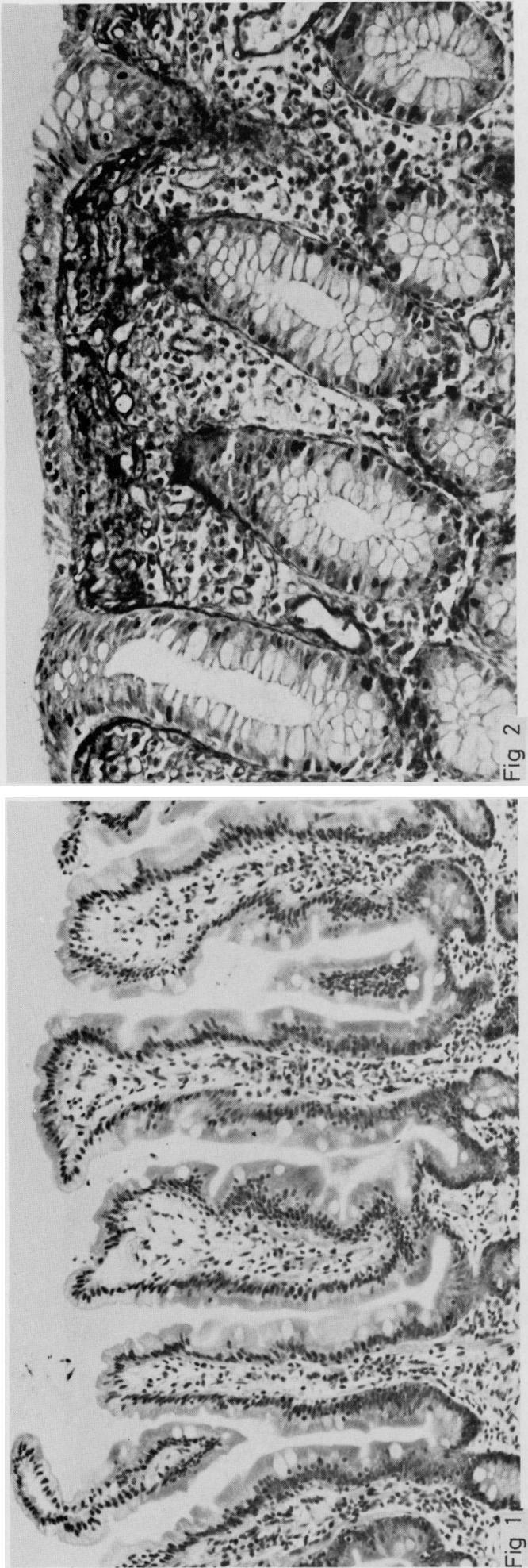

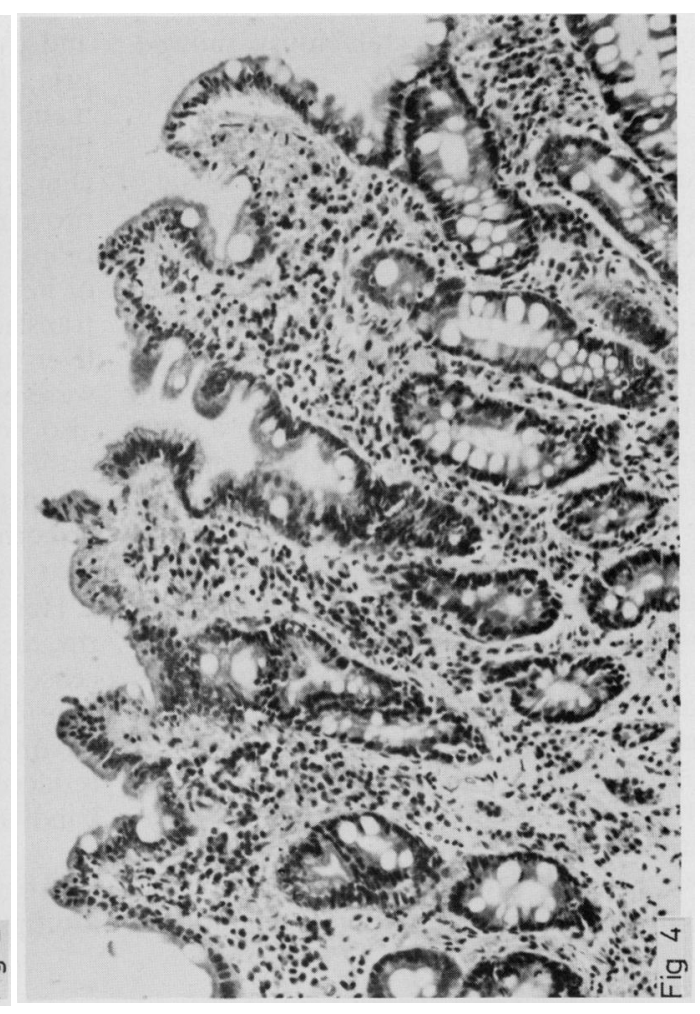

25

1.

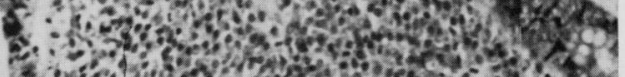

2 ont

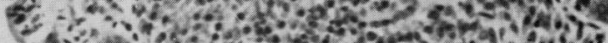

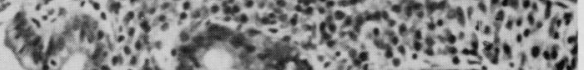

and

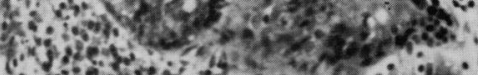

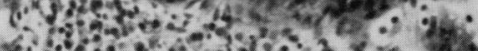

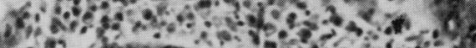

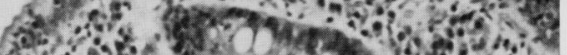

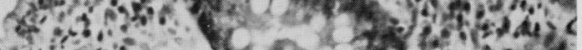
Geve

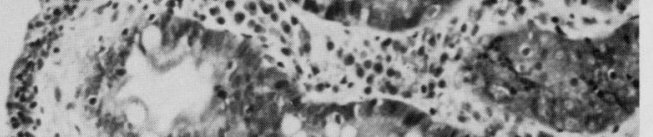

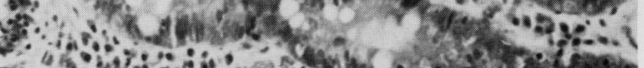

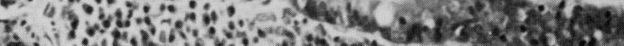

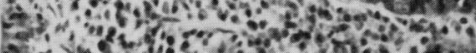

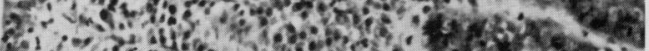

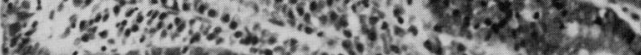

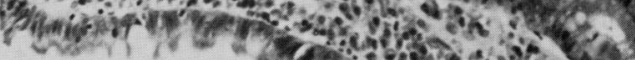

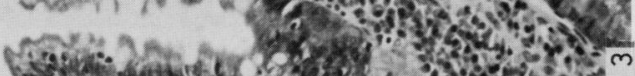

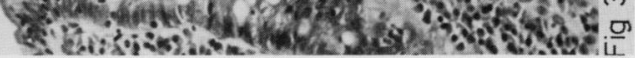


she was asymptomatic, and rectal biopsy showed normal histological appearances.

CASE 2

A 53 year old woman presented in 1983 with a 12 month history of diarrhoea. Physical examination and sigmoidoscopy were normal but rectal biopsy showed a mild, non-specific proctitis. No pathogens or parasites were isolated from her stools, full blood count, renal, liver and thyroid function tests were normal but jejunal biopsy was abnormal, with subtotal villous atrophy, crypt hyperplasia, an increased intra-epithelial lymphocyte count and a chronic inflammatory infiltrate in the lamina propria. There was patchy thickening of the small intestinal basement membrane (Fig. 3). Small bowel barium enema was normal. Treatment with a gluten free diet had no effect on her symptoms, and jejunal mucosal morphology was unchanged after two months treatment. She was started on treatment with prednisolone $30 \mathrm{mg}$ daily and her diarrhoea improved. After withdrawal of steroids her diarrhoea recurred, and jejunal biopsy taken three months after cessation of steroid treatment showed partial villous atrophy with crypt hyperplasia and increased numbers of intra-epithelial lymphocytes and chronic inflammatory cells in the lamina propria. Thickening of the small intestinal basement membrane was less marked than in the previous biopsies. Mucosal morphology on this occasion (Fig. 4) was considered to show some evidence of improvement when compared with her initial jejunal biopsy. She was admitted for further investigation of her diarrhoea. Full blood count, serum iron and transferrin, folate and $B_{12}$, urea and electrolytes, liver function tests, immunoglobulins and proteins were normal. Small and large bowel enemata were also normal, as was colonoscopy, but colonic biopsies showed a sub-epithelial band of collagen compatible with collagenous colitis (Fig. 5). ${ }^{14} \mathrm{C}$ glycocholate breath test and pancreatic function tests were normal, and faecal fat was not raised.

Her symptoms also failed to respond to salazopyrin, metronidazole, or rectal steroids, but diarrhoea ceased within two weeks of treatment with prednisolone $30 \mathrm{mg}$ daily. Colonic biopsies from splenic flexure, descending and sigmoid colon showed a reduced inflammatory infiltrate with a persistent band of subepithelial collagen.

HISTOLOGY OF COLONIC BIOPSIES

Multiple biopsies were taken from various sites in

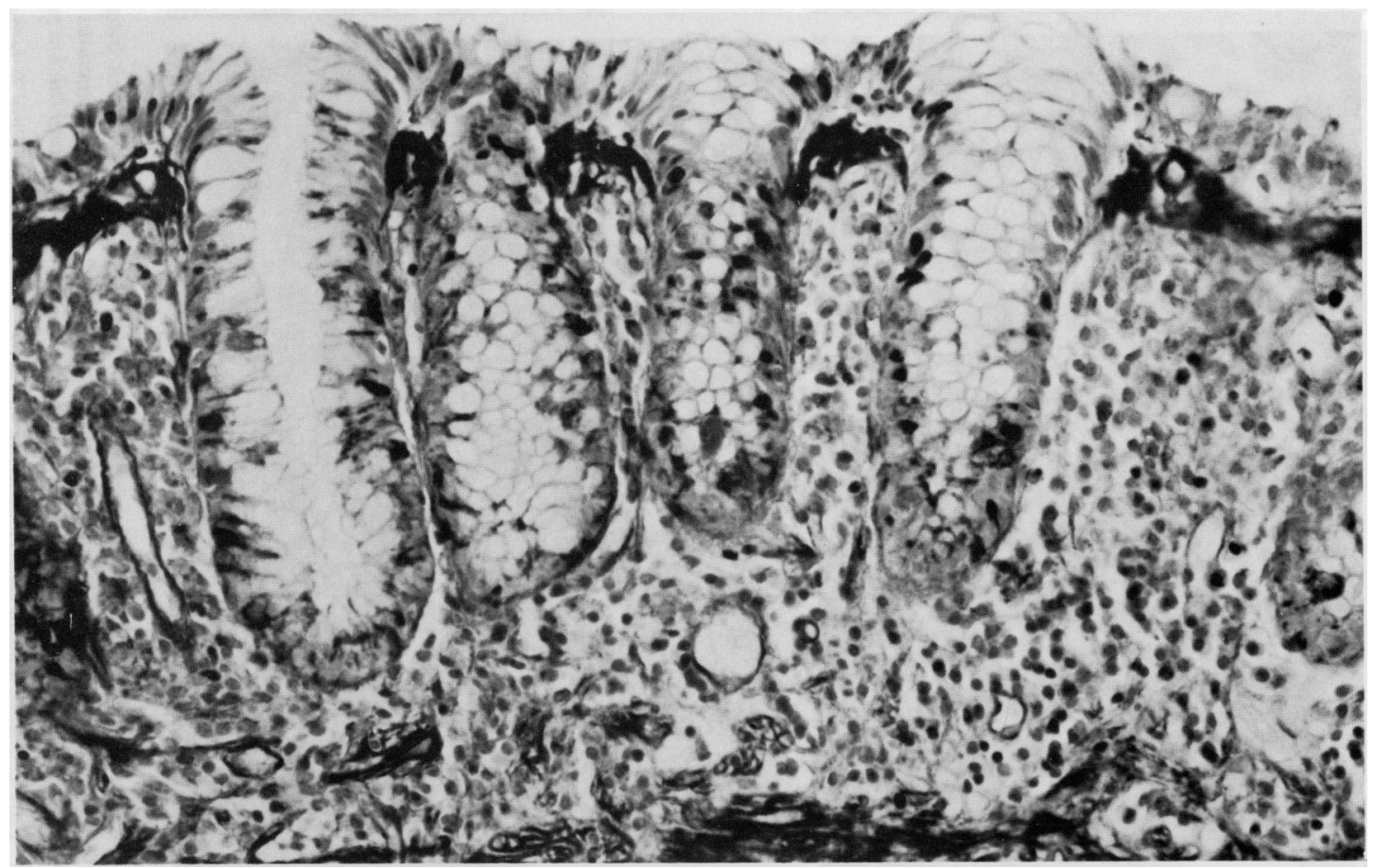

Fig. 5 Case 2: Colonic biopsy (ascending colon) with dark staining thickened collagenous band under the basement membrane. Mean band thickness is 24 um (Trichrome). 
Table Thickness of subepithelial collagen band and degree of colonic inflammation at various sites in the colon

\begin{tabular}{|c|c|c|c|c|}
\hline Site & $\begin{array}{l}\text { Case } 1 \\
\text { Mean collagen band } \\
\text { thickness }(\mu m \pm S D)\end{array}$ & $\begin{array}{l}\text { Inflammation } \\
\text { in lamina propria }\end{array}$ & $\begin{array}{l}\text { Case } 2 \\
\text { Mean collagen band } \\
\text { thickness }(\mu m \pm S D)\end{array}$ & $\begin{array}{l}\text { Inflammation } \\
\text { in lamina propria }\end{array}$ \\
\hline Ascending colon & $22 \pm 8$ & Mild & $24 \pm 9$ & Moderate \\
\hline Transverse colon & $25 \pm 5$ & Mild & $22 \pm 4$ & Moderatc \\
\hline Descending colon & $17 \pm 5$ & Mild & $26 \pm 7$ & Mild \\
\hline Sigmoid colon & $34 \pm 9$ & Mild & $9 \pm 3$ & Moderate \\
\hline Rectum & $13 \pm 2$ & Nil & $10 \pm 3$ & Moderate \\
\hline
\end{tabular}

the colon from both patients, and fixed in 10\% buffered formalin solution. Paraffin sections were stained with haematoxylin and eosin ( $H \& E)$, van Gieson and Congo Red stains. Thickness of the subepithlial collagenous band was measured on van Gieson stained sections, using a micrometer eyepiece and a $\times 25$ objective, and the mean thickness calculated from 20 point counts on each section. Mucosal inflammation was graded as mild, moderate, or severe. Histological appearances were similar in all biopsies from both patients. A dense, eosinophilic band immediately beneath the colonic surface epithelium could be seen on $\mathrm{H} \& \mathrm{E}$ stained sections (Figs. 2, 5), which was not stained with Congo Red but stained positively for collagen with van Gieson. The collagen band was confined to the intercryptal area and did not extend deep into the pericryptal area. The mucosal crypts were regular and the muscularis mucosae and submucosal vessels appeared normal. In one biopsy there was no histological evidence of inflammation, but in all others there was a mild to moderate infiltrate of mononuclear cells, including plasma cells, in the lamina propria.

The thickness of the collagen band and degree of mucosal inflammation at each site of the colon are shown in the Table. There is significant thickening of the basement membrane throughout the colon in both patients, with a tendency for the collagen deposition to be less marked in the distal colon.

Immunoperoxidase techniques revealed no evidence of deposition of IgG, IgA, IgM or complement $\left(\mathrm{C}_{3}\right)$ within the thickened collagenous band in either patient.

\section{Discussion}

Previous reports have established that collagenous colitis is a discrete entity, occurring predominantly in middle aged and elderly women ${ }^{5}$ in which watery diarrhoea is the result of active colonic secretion of chloride associated with passive transfer of sodium and water into the colonic lumen. ${ }^{8}$
Collagenous colitis is diagnosed by histological examination of colonic biopsies, which shows the characteristic band of collagen deposited beneath the epithelial basement membrane, usually associated with a non-specific chronic inflammatory infiltrate. The relationship between the inflammatory reaction, the collagen deposition and the development of symptoms is uncertain, and the stimulus to collagen deposition is also unknown. Collagenous colitis can be distinguished from the collagen deposition secondary to other colonic diseases by the absence of features of inflammatory or neoplastic disease. ${ }^{3}$ Significant basement membrane thickening (defined as a thickness in excess of $10 \mu \mathrm{m}$ ) is rare in association with other colonic pathology. ${ }^{3}$ The collagenous band is confined to the intercryptal areas, and does not extend deep into the pericryptal areas.

Previous reports of collagenous colitis have described response to treatment with antidiarrhoeals, ${ }^{8} 9$ mepacrine ${ }^{11}$ metronidazole, ${ }^{12}$ salazopyrin $^{13}$ and steroids. ${ }^{14}$ Spontaneous resolution of symptoms, with disappearance of the collagenous band, has also been described, however, ${ }^{15}$ and it is unclear whether symptoms are related to the presence of microscopic inflammation, or to the presence of the subepithelial collagen. The resolution of symptoms despite persistence of the collagen band we describe in one case suggests that symptoms may be more closely related to the chronic colonic inflammation.

The histological features of the colonic biopsies in these two cases are similar to those previously described in collagenous colitis, but these patients are unique in the coexistence of small intestinal villous atrophy. The diagnosis of coeliac disease is established in case 1 by the excellent clinical response to a gluten free diet, and by the preservation of normal jejunal morphology on treatment, although gluten challenge has never been performed. The diagnosis of coeliac disease in case 2 is not established, since there was no clinical or histological response to gluten withdrawal. Steroid 
treatment resulted in temporary improvement in her symptoms and the appearances of the jejunal mucosa after cessation of steroid therapy do show some evidence of morphological improvement when compared with her initial biopsy. We have not shown any other cause for villous atrophy and therefore believe her to represent non-responsive coeliac disease.

Subepithelial collagen deposition within the lamina propria of the small intestine is a feature of coeliac disease, which usually remits on treatment with a gluten free diet, as other aspects of the mucosal morphology also improve. ${ }^{1}$ It has been suggested that such collagen deposition is the result of a chronic subepithelial inflammatory reaction, possibly related to immune complex deposition because of continued gluten ingestion. Immune complex deposition has not been observed in the colon of patients with collagenous colitis. ${ }^{4} 101314$ In both of the cases we describe collagenous colitis developed during treatment with a strictly observed gluten free diet and is, therefore, unlikely to represent a manifestation of gluten toxicity to the colon.

Diarrhoea in coeliac disease which persists despite treatment with a gluten free diet may be caused by associated intestinal malignancy, pancreatic insufficiency, infestation or idiopathic inflammatory bowel disease. The relationship between inflammatory bowel disease, microscopic colitis and collagenous colitis is uncertain.

Further investigation of the frequency of collagenous colitis in coeliac disease and of the relationship between small intestinal and colonic collagen deposition is indicated. We propose that collagenous colitis should be considered as a further cause for failure of diarrhoeal symptoms in coeliac disease to respond to a gluten free diet.

We are grateful to Mr R Fawkes, Department of Pathology, Ninewells Hospital and Medical School, University of Dundee, for preparation of the photomicrographs, and to Ms M Hughes for typing the manuscript.

\section{References}

1 Bossart R, Henry K, Booth CC, Doe WF. Subepithelial collagen in intestinal malabsorption. Gut 1975; 16: 18-22.

2 Flejou JF, Grimaud JA, Molas G, Baviera E, Potet F. Collagenous colitis. Ultrastructural study and collagen immunotyping of four cases. Arch Pathol Lab Med 1984; 108: 977-82.

3 Gledhill A, Cole FM. Significance of basement membrane thickening in the human colon. Gut 1984; 25: 1085-8.

4 Teglbjaers PS, Thaysen EH. Collagenous colitis: an ultrastructural study of a case. Gastroenterology 1982; 82: $561-3$.

5 van der Gord JJ, Geboes K, Desmet VJ. Collagenous colitis: an abnormal collagen table? Two new cases and review of the literature. Am J Gastroenterol 1982; 77: 377-81.

6 Colina F, Solis-Herruzo JA, Munoz-Yasue MT, Vazquez G, Perez-Barrios A. Collagenous colitis: the clinical and morphological features. Postgrad Med J 1982; 58: 390-5.

7 Guarda LA, Nelson RS, Stroehlein JR, Korinek JK, Raymond AK. Collagenous colitis. Am J Clin Pathol 1983; 80: 503-7.

8 Rask-Madsen J, Grove O, Hansen MG, Bukhave K, Soient C, Henrik-Nielsen R. Colonic transport of water and electrolytes in a patient with secretory diarrhoea due to collagenous colitis. Dig Dis Sci 1983; 28: 1141-6.

9 Eaves ER, Wallis PL, McIntyre RL, Korman MG. Collagenous colitis: a recently recognised reversible clinicopathological entity. Aust NZ J Med 1983; 13: 630-2.

10 Fausa O, Foerster A, Hovis T. Collagenous colitis. A clinical, histological and ultrastructural study. Scand $J$ Gastroenterol 1985; suppl 107: 8-23.

11 Pieterse AS, Hecker R, Rowland R. Collagenous colitis: a distinctive and potentially reversible disorder. J Clin Pathol 1982; 35: 338-40.

12 Mosensen AM, Olsen JH, Gudmand-Hoyer E. Collagenous colitis. Acta Medica Scand 1984; 216: 535-40.

13 Farah DA, Mills PR, Lee FD, McLay A, Russell RI. Collagenous colitis: possible response to sulfasalazine and local steroid therapy. Gastroenterology 1985; 88: 792-7.

14 Teglbjaerg PS, Thaysen EH, Jensen HH. Development of collagenous colitis in sequential biopsy specimens. Gastroenterology 1984; 87: 703-9.

15 Debonanic JC, De Galocsy C, Caholessur MO, Haot J. Collagenous colitis: a transient condition? Report of two cases. Dis Colon Rectum 1984; 27: 672-6. 